\title{
A nonconforming finite element method for the Stokes equations using the Crouzeix-Raviart element for the velocity and the standard linear element for the pressure
}

\author{
Bishnu P. Lamichhane*
}

June 27, 2013

\begin{abstract}
We present a finite element method for Stokes equations using the Crouzeix-Raviart element for the velocity and the continuous linear element for the pressure. We show that the inf-sup condition is satisfied for this pair. Two numerical experiments are presented to support the theoretical results.
\end{abstract}

Keywords: Stokes equations, mixed finite elements, Crouzeix-Raviart element, nonconforming method, inf-sup condition

AMS Subject Classification: 65N30, 65N15

\section{Introduction}

The finite element method is very popular method for approximating the solutions of partial differential equations. There are many finite element methods for Stokes and Navier-Stokes equations leading to optimal convergence [7, 3, 2. However, the search for simple and efficient finite element schemes for Stokes and Navier-Stokes equations is still an active area of research. The nonconforming techniques and discontinuous Galerking methods have also gained high popularity. In particular, it is easier to prove the inf-sup condition with the nonconforming methods, and they are also quite simple

${ }^{*}$ School of Mathematical \& Physical Sciences, Mathematics Building, University of Newcastle, University Drive, Callaghan, NSW 2308, Australia, Bishnu.Lamichhane@newcastle.edu.au 
to implement. Moreover, nonconforming finite element basis functions have smaller support compared to conforming finite elements. One of the most popular nonconforming finite element methods is the method based on discretizing the velocity with the lowest order Crouzeix-Raviart element and the pressure with the piecewise constant functions [5]. Recently a stabilized finite element method is presented using again Crouzeix-Raviart element for discretizing the velocity but replacing the piecewise constant pressure element with the continuous piecewise linear pressure [10]. We want to emphasize two benefits of using the continuous linear pressure over the discontinuous piecewise constant pressure. With the continuous pressure we expect to get a better approximation for the pressure. Although we could not prove this better approximation theoretically, numerical experiments support this argument. The second advantage is that it is easy to visualize the continuous pressure than the discontinuous pressure.

In this contribution, we show that the stabilization proposed in [10] is unnecessary. In fact, we show that the finite element pair - the lowest order Crouzeix-Raviart element for the velocity and the continuous linear element for the pressure - yields a stable approximation scheme. The proof is based on using an interpolation operator satisfying certain conditions. The proof also establishes that for a low order finite element method based on simplicial meshes with a piecewise constant pressure, the piecewise constant pressure can be replaced by the continuous linear pressure. The rest of the paper is organized as follows. In the next section we recall the Stokes equations, and we prove our main results in Section 3. Two numerical experiments are presented in 4 to support the theoretical result. Finally, a conclusion is drawn in Section 5 .

\section{Stokes equations}

This section is devoted to the introduction of the boundary value problem of the Stokes equations. Let $\Omega$ in $\mathbb{R}^{d}, d \in\{2,3\}$, be a bounded domain with polygonal or polyhedral boundary $\Gamma$. For a prescribed body force $\boldsymbol{f} \in\left[L^{2}(\Omega)\right]^{d}$, the Stokes equations with homogeneous Dirichlet boundary condition in $\Gamma$ reads

$$
\begin{aligned}
& -\nu \Delta \boldsymbol{u}+\nabla p=\boldsymbol{f} \text { in } \Omega \\
& \operatorname{div} \boldsymbol{u}=0 \text { in } \Omega
\end{aligned}
$$

with $\boldsymbol{u}=\mathbf{0}$ on $\Gamma$, where $\boldsymbol{u}$ is the velocity, $p$ is the pressure, and $\nu$ denotes the viscosity of the fluid. 
Here we use standard notations $L^{2}(\Omega), H^{1}(\Omega)$ and $H_{0}^{1}(\Omega)$ for Sobolev spaces, see [2, 4] for details. Let $\boldsymbol{V}:=\left[H_{0}^{1}(\Omega)\right]^{d}$ be the vector Sobolev space with inner product $(\cdot, \cdot)_{1, \Omega}$ and norm $\|\cdot\|_{1, \Omega}$ defined in the standard way: $(\boldsymbol{u}, \boldsymbol{v})_{1, \Omega}:=\sum_{i=1}^{d}\left(u_{i}, v_{i}\right)_{1, \Omega}$, and the norm being induced by this inner product. We also define another subspace $M$ of $L^{2}(\Omega)$ as

$$
M=\left\{q \in L^{2}(\Omega): \int_{\Omega} q d x=0\right\} .
$$

The weak formulation of the Stokes equations is to find $(\boldsymbol{u}, p) \in \boldsymbol{V} \times M$ such that

$$
\begin{gathered}
\nu \int_{\Omega} \nabla \boldsymbol{u}: \nabla \boldsymbol{v} d x+\int_{\Omega} \operatorname{div} v p d x=\ell(\boldsymbol{v}), \quad \boldsymbol{v} \in \boldsymbol{V} \\
\int_{\Omega} \operatorname{div} \boldsymbol{u} q d x \\
=0, \quad q \in M
\end{gathered}
$$

where $\ell(\boldsymbol{v})=\int_{\Omega} \boldsymbol{f} \cdot \boldsymbol{v} d x$. It is well-known that the weak formulation of the Stokes problem is well-posed [7]. In fact, if the domain $\Omega$ is convex, and $\boldsymbol{f} \in\left[L^{2}(\Omega)\right]^{d}$, we have $\boldsymbol{u} \in\left[H^{2}(\Omega)\right]^{d}, p \in H^{1}(\Omega)$ and the a priori estimate holds

$$
\|\boldsymbol{u}\|_{2, \Omega}+\|p\|_{1, \Omega} \leq C\|\boldsymbol{f}\|_{0, \Omega},
$$

where the constant $C$ depends on the domain $\Omega$.

\section{$3 \quad$ Finite element discretizations}

We consider a quasi-uniform triangulation $\mathcal{T}_{h}$ of the polygonal or polyhedral domain $\Omega$, where $\mathcal{T}_{h}$ consists of simplices, either triangles or tetrahedra, where $h$ denotes the mesh-size. Note that $\mathcal{T}_{h}$ denotes the set of elements. For an element $T \in \mathcal{T}_{h}$, let $P_{n}(T)$ be the set of all polynominals of degree less than or equal to $n \in \mathbb{N} \cup\{0\}$. The the set of all vertices in $\mathcal{T}_{h}$ is denoted by $\mathcal{V}_{h}:=\left\{\boldsymbol{x}_{i}\right\}_{i=1}^{N}$, and $\mathcal{N}_{h}:=\{1,2, \cdots, N\}$.

Let $\mathcal{E}_{h}$ be the set of all edges in two dimensions and faces in three dimensions. The continuity of a function $\boldsymbol{v}_{h}$ across an edge or a face $e \in \mathcal{E}_{h}$ for the nonconforming finite element will be enforced according to

$$
J_{e}\left(\boldsymbol{v}_{h}\right):=\int_{e}\left[\boldsymbol{v}_{h}\right]_{e} d \sigma=0
$$

where $\left[\boldsymbol{v}_{h}\right]_{e}$ is the jump of the function $\boldsymbol{v}_{h}$ across the edge $e$. Then the Crouzeix-Raviart finite element space $\boldsymbol{V}_{h}$ is defined as

$$
\boldsymbol{W}_{h}:=\left\{\boldsymbol{v}_{h} \in\left[L^{2}(\Omega)\right]^{d}:\left.\boldsymbol{v}_{h}\right|_{K} \in\left[P_{1}(K)\right]^{d}, K \in \mathcal{T}_{h}, J_{e}\left(\boldsymbol{v}_{h}\right)=0, e \in \mathcal{E}_{h}\right\} .
$$


The finite element basis functions of $\boldsymbol{W}_{h}$ are associated with the mid-points of the edges of triangles or the bary-centers of the faces of tetrahedra. To impose the homogeneous Dirichlet boundary condition on $\Gamma$ we define $\boldsymbol{V}_{h}$ as a subset of $\boldsymbol{W}_{h}$ where

$$
\boldsymbol{V}_{h}:=\left\{\boldsymbol{v}_{h} \in \boldsymbol{W}_{h}: \int_{e} \boldsymbol{v}_{h} d \sigma=0, e \in \mathcal{E}_{h} \cap \Gamma\right\} .
$$

As $\boldsymbol{V}_{h} \not \subset \boldsymbol{V}$, we cannot use the standard $H^{1}$-norm for an element in $\boldsymbol{V}_{h}$. So we define a broken $H^{1}$ - norm on $\boldsymbol{V}_{h}$ as

$$
\left\|\boldsymbol{v}_{h}\right\|_{1, h}:=\sqrt{\sum_{T \in \mathcal{T}_{h}}\left\|\boldsymbol{v}_{h}\right\|_{1, T}^{2}}, \quad \boldsymbol{v}_{h} \in \boldsymbol{V}_{h}
$$

The broken $H^{1}$ - semi-norm is similarly defined

$$
\left|\boldsymbol{v}_{h}\right|_{1, h}:=\sqrt{\sum_{T \in \mathcal{T}_{h}}\left|\boldsymbol{v}_{h}\right|_{1, T}^{2}}, \quad \boldsymbol{v}_{h} \in \boldsymbol{V}_{h} .
$$

We also define two relevant finite element spaces

$$
Q_{h}:=\left\{q_{h} \in L^{2}(\Omega):\left.q_{h}\right|_{K}=P_{0}(K), K \in \mathcal{T}_{h}, \int_{\Omega} q_{h} d x=0\right\},
$$

and

$$
P_{h}:=\left\{q_{h} \in H^{1}(\Omega):\left.q_{h}\right|_{K}=P_{1}(K), K \in \mathcal{T}_{h}, \int_{\Omega} q_{h} d x=0\right\}
$$

Now our discrete weak formulation of Stokes equations can be written as: find $\left(\boldsymbol{u}_{h}, p_{h}\right) \in \boldsymbol{V}_{h} \times P_{h}$ such that

$$
\begin{aligned}
a\left(\boldsymbol{u}_{h}, \boldsymbol{v}_{h}\right)+b\left(\boldsymbol{v}_{h}, p_{h}\right) & =\ell\left(\boldsymbol{v}_{h}\right), & & \boldsymbol{v}_{h} \in \boldsymbol{V}_{h}, \\
b\left(\boldsymbol{u}_{h}, q_{h}\right) & =0, & & q_{h} \in P_{h},
\end{aligned}
$$

where

$$
a\left(\boldsymbol{u}_{h}, \boldsymbol{v}_{h}\right):=\nu \sum_{T \in \mathcal{T}_{h}} \int_{T} \nabla \boldsymbol{u}_{h}: \nabla \boldsymbol{v}_{h} d x, b\left(\boldsymbol{v}_{h}, q_{h}\right):=\sum_{T \in \mathcal{T}_{h}} \int_{T} \operatorname{div} \boldsymbol{v}_{h} q_{h} d x
$$

In order to show that the saddle point problem (3) has a unique solution, we want to apply a standard saddle point theory [3, 2]. To this end, we need to show the following three conditions of well-posedness. 
1. The linear form $\ell(\cdot)$, the bilinear forms $a(\cdot, \cdot)$ and $b(\cdot, \cdot)$ are continuous on the spaces on which they are defined.

2. The bilinear form $a(\cdot, \cdot)$ is coercive on the space $K$ defined as

$$
K=\left\{\boldsymbol{v}_{h} \in \boldsymbol{V}_{h}: b\left(\boldsymbol{v}_{h}, q_{h}\right)=0, q_{h} \in P_{h}\right\}
$$

3. The finite element pair $\left(\boldsymbol{V}_{h}, P_{h}\right)$ satisfies a uniform inf-sup condition. That means the bilinear form $b(\cdot, \cdot)$ satisfies

$$
\sup _{\boldsymbol{v}_{h} \in \boldsymbol{V}_{h}} \frac{b\left(\boldsymbol{v}_{h}, q_{h}\right)}{\left\|\boldsymbol{v}_{h}\right\|_{1, h}} \geq \beta\left\|q_{h}\right\|_{0, \Omega}, \quad q_{h} \in P_{h}
$$

for a constant $\beta>0$ independent of the mesh-size $h$.

It is standard that the linear form $\ell(\cdot)$, and the bilinear forms $a(\cdot, \cdot)$ and $b(\cdot, \cdot)$ are continuous, and $a(\cdot, \cdot)$ is coercive due to Poincaré inequality. It remains to prove that the finite element pair $\left(\boldsymbol{V}_{h}, P_{h}\right)$ satisfies the uniform inf-sup condition.

A finite element method for the Stokes equations is presented in [10] using the finite element pair $\left(\boldsymbol{V}_{h}, P_{h}\right)$, where the authors use a local stabilization to obtain the stability of the system. Here we show that the stabilization is not necessary, and the finite element pair $\left(\boldsymbol{V}_{h}, P_{h}\right)$ also satisfies the uniform inf-sup condition. This is the main goal of the paper.

To show this we use the fact that the finite element pair $\left(\boldsymbol{V}_{h}, Q_{h}\right)$ satisfies a uniform inf-sup condition, which is proved by Crouzeix and Raviart [5]. Hence there exists a constant $\hat{\beta}>0$ indpendent of the mesh-size $h$ such that

$$
\sup _{\boldsymbol{v}_{h} \in \boldsymbol{V}_{h}} \frac{b\left(\boldsymbol{v}_{h} q_{h}\right)}{\left\|\boldsymbol{v}_{h}\right\|_{1, h}} \geq \hat{\beta}\left\|q_{h}\right\|_{0, \Omega}, \quad q_{h} \in Q_{h} .
$$

We now introduce another finite element space

$$
R_{h}:=\left\{q_{h} \in Q_{h}:\left.q_{h}\right|_{S_{i}}=P_{0}\left(S_{i}\right), i \in \mathcal{N}_{h}\right\},
$$

where $S_{i}$ is the support of the standard linear basis function $\phi_{i} \in P_{h}$ associated with the vertex $\boldsymbol{x}_{i} \in \mathcal{V}_{h}$. We use this space to show that the finite element pair $\left(\boldsymbol{V}_{h}, P_{h}\right)$ satisfies the uniform inf-sup condition. Since $R_{h} \subset Q_{h}$, the finite element pair $\left(\boldsymbol{V}_{h}, R_{h}\right)$ also satisfies the uniform inf-sup condition. Thus we can get a consant $\tilde{\beta}>0$ independent of the mesh-size $h$ such that

$$
\sup _{\boldsymbol{v}_{h} \in \boldsymbol{V}_{h}} \frac{b\left(\boldsymbol{v}_{h}, q_{h}\right)}{\left\|\boldsymbol{v}_{h}\right\|_{1, h}} \geq \tilde{\beta}\left\|q_{h}\right\|_{0, \Omega}, \quad q_{h} \in R_{h} .
$$


In order to show that the finite element pair $\left(\boldsymbol{V}_{h}, P_{h}\right)$ satisfies the uniform inf-sup condition we introduce an interpolation operator $I_{h}$ [6, 8, 9]

$$
I_{h}: P_{h} \rightarrow R_{h}
$$

defined as

$$
I_{h} q_{h}=\sum_{i=1}^{N} q_{i} \chi_{i}
$$

for $q_{h}=\sum_{i=1}^{N} q_{i} \phi_{i} \in P_{h}$, where $\chi_{i}$ is a characteristic function of the set $S_{i}$, and $\phi_{i}$ is the standard linear hat function associated with the vertex $\boldsymbol{x}_{i}$ for $i \in \mathcal{N}_{h}$. We note that the interpolation operator $I_{h}$ is well-defined, and it is not the standard Fortin interpolation operator. We define an element-wise defined divergence $\operatorname{div}_{\mathrm{h}} \boldsymbol{v}_{h}$ of a vector function $\boldsymbol{v}_{h} \in \boldsymbol{V}_{h}$

$$
\left.\operatorname{div}_{\mathrm{h}} \boldsymbol{v}_{h}\right|_{T}=\left.\operatorname{div} \boldsymbol{v}_{h}\right|_{T}, \quad T \in \mathcal{T}_{h} .
$$

We have now following two lemmas. In the following, we use generic constants $C, C_{1}$ and $C_{2}$. They may take different values at different places but they do not depend on the mesh-size $h$.

Lemma 1 Given $\boldsymbol{v}_{h} \in \boldsymbol{V}_{h}$ and $q_{h} \in P_{h}$ we have

$$
(d+1) \int_{\Omega} \operatorname{div}_{\mathrm{h}} \boldsymbol{v}_{h} q_{h} d x=\int_{\Omega} \operatorname{div}_{\mathrm{h}} \boldsymbol{v}_{h} I_{h} q_{h} d x .
$$

Proof Let $q_{h}=\sum_{i=1}^{N} q_{i} \phi_{i}$, and hence $I_{h} q_{h}=\sum_{i=1}^{N} q_{i} \chi_{i}$. Then using the fact that $\operatorname{div}_{\mathrm{h}} \boldsymbol{v}_{h}$ is piecewise constant with respect to the mesh $\mathcal{T}_{h}$ we have

$$
\int_{\Omega} \operatorname{div}_{\mathrm{h}} \boldsymbol{v}_{h} I_{h} q_{h} d x=\sum_{i=1}^{N} q_{i} \int_{S_{i}} \operatorname{div}_{\mathrm{h}} \boldsymbol{v}_{h} d x=\left.\sum_{i=1}^{N} q_{i} \sum_{T \subset S_{i}}|T|\left(\operatorname{div} \boldsymbol{v}_{h}\right)\right|_{T} .
$$

Similarly,

$$
\int_{\Omega} \operatorname{div}_{\mathrm{h}} \boldsymbol{v}_{h} q_{h} d x=\sum_{i=1}^{N} q_{i} \int_{S_{i}} \operatorname{div}_{\mathrm{h}} \boldsymbol{v}_{h} \phi_{i} d x=\left.\frac{1}{d+1} \sum_{i=1}^{N} q_{i} \sum_{T \subset S_{i}}|T|\left(\operatorname{div} \boldsymbol{v}_{h}\right)\right|_{T} .
$$

Lemma 2 There exist positive constants $C_{1}$ and $C_{2}$ with

$$
C_{1}\left\|q_{h}\right\|_{0, \Omega} \leq\left\|I_{h} q_{h}\right\|_{0, \Omega} \leq C_{2}\left\|q_{h}\right\|_{0, \Omega}
$$


Proof The proof follows by using the fact that $\left\|I_{h} q_{h}\right\|_{0}^{2}$ and $\left\|q_{h}\right\|_{0}^{2}$ and $\sum_{i=1}^{N} q_{i}^{2} h_{i}^{2}$ are equivalent, where $h_{i}$ is the local mesh-size at the $i$-th node of $\mathcal{T}_{h}$.

We are now in a position to prove the main result of the paper.

Theorem 3 The finite element pair $\left(\boldsymbol{V}_{h}, P_{h}\right)$ satisfies the inf-sup condition. That means there exists a consant $\beta>0$ independent of mesh-size $h$ such that

$$
\sup _{\boldsymbol{v}_{h} \in \boldsymbol{V}_{h}} \frac{b\left(\boldsymbol{v}_{h}, q_{h}\right)}{\left\|\boldsymbol{v}_{h}\right\|_{1, h}} \geq \beta\left\|q_{h}\right\|_{0, \Omega}, \quad q_{h} \in P_{h} .
$$

Proof The proof is straightforward. Let $q_{h} \in P_{h}$, then $I_{h} q_{h} \in R_{h}$. Since the pair $\left(\boldsymbol{V}_{h}, R_{h}\right)$ satisfies the inf-sup condition, we can find an element $\boldsymbol{v}_{h} \in \boldsymbol{V}_{h}$ satisfying

$$
\int_{\Omega} \operatorname{div}_{\mathrm{h}} \boldsymbol{v}_{h} I_{h} q_{h} d x \geq C\left\|I_{h} q_{h}\right\|_{0, \Omega}^{2}, \quad\left\|\boldsymbol{v}_{h}\right\|_{1, h} \leq C\left\|I_{h} q_{h}\right\|_{0, \Omega} .
$$

Hence the results of Lemma 1 and 2 yield

$$
\int_{\Omega} \operatorname{div}_{\mathrm{h}} \boldsymbol{v}_{h} I_{h} q_{h} d x=(d+1) \int_{\Omega} \operatorname{div}_{\mathrm{h}} \boldsymbol{v}_{h} q_{h} d x \geq C\left\|I_{h} q_{h}\right\|_{0, \Omega}^{2} \geq C\left\|q_{h}\right\|_{0, \Omega}^{2},
$$

and $\left\|\boldsymbol{v}_{h}\right\|_{1, h} \leq C\left\|q_{h}\right\|_{0, \Omega}$. Hence we have a constant $C$ independent of the mesh-size $h$ such that

$$
\sup _{\boldsymbol{v}_{h} \in \boldsymbol{V}_{h}} \frac{b\left(\boldsymbol{v}_{h}, q_{h}\right)}{\left\|\boldsymbol{v}_{h}\right\|_{1, h}} \geq C\left\|q_{h}\right\|_{0, \Omega}, \quad q_{h} \in P_{h} .
$$

\section{$4 \quad$ Numerical experiments}

In this section we present two numerical experiments for the proposed finite element scheme. For the numerical experiment we consider a simple unit square $\Omega=(0,1)^{2}$, and a uniform initial triangulation consisting of eight triangles.

First example. For the first example we consider an exact solution given in [1], where the exact solution for the velocity $\boldsymbol{u}=\left(u_{1}, u_{2}\right)$ is given by

$u_{1}=x+x^{2}-2 x y+x^{3}-3 x y^{2}+x^{2} y, \quad u_{2}=-y-2 x y+y^{2}-3 x^{2} y+y^{3}-x y^{2}$, 
and the exact solution for the pressure is given by

$$
p=x y+x+y+x^{3} y^{2}-\frac{4}{3} .
$$

Using the kinematic viscosity $\nu=1$ and the exact solution we compute the right-hand side function $f$ and the Dirichlet boundary condition for the velocity. Here we compute the errors in the velocity and the pressure approximation using the broken $H^{1}$ - semi-norm and the $L^{2}$ - norm, respectively. The numerical results are tabulated in Table 1. From the presented table we can see the optimal convergence of the velocity approximation in the broken $H^{1}$ - semi-norm, and a super-convergence result for the pressure in the $L^{2}$-norm. As we expect a convergence rate of order 1 for the pressure approximation in the $L^{2}$-norm but get a better approximation of order 1.5 , this is a super-convergence. This better convergence is due to the fact that we have used the standard continuous linear finite element space for the pressure approximation.

Table 1: Discretization errors for the velocity and pressure, Example 1

\begin{tabular}{|c|c|c|c|c|c|}
\hline level $l$ & \# elem. & \multicolumn{2}{|c|}{$\left|u-u_{h}\right|_{1, h}$} & \multicolumn{2}{c|}{$\left\|p-p_{h}\right\|_{0, \Omega}$} \\
\hline 0 & 8 & $2.53070 \mathrm{e}-01$ & & $9.75595 \mathrm{e}-02$ & \\
\hline 1 & 64 & $1.32989 \mathrm{e}-01$ & 0.93 & $3.35584 \mathrm{e}-02$ & 1.54 \\
\hline 2 & 512 & $6.78573 \mathrm{e}-02$ & 0.97 & $1.14072 \mathrm{e}-02$ & 1.56 \\
\hline 3 & 4096 & $3.42262 \mathrm{e}-02$ & 0.99 & $3.87893 \mathrm{e}-03$ & 1.56 \\
\hline 4 & 32768 & $1.71804 \mathrm{e}-02$ & 0.99 & $1.32844 \mathrm{e}-03$ & 1.55 \\
\hline 5 & 262144 & $8.60590 \mathrm{e}-03$ & 1.00 & $4.58976 \mathrm{e}-04$ & 1.53 \\
\hline
\end{tabular}

Second example. For our second example we consider the same computational domain but a different exact solution with kinematic viscosity $\nu=5$. Here the exact solution for the velocity $\boldsymbol{u}=\left(u_{1}, u_{2}\right)$ is given by

$$
u_{1}=\mathrm{e}^{-x+y} \sin (5 x), \quad u_{2}=\mathrm{e}^{-x+y} \sin (5 x)-5 \mathrm{e}^{-x+y} \cos (5 x),
$$

and the exact solution for the pressure is given by

$$
p=x y(1-x)(1-y)-\frac{1}{36} \text {. }
$$

As in the first example, the source term $\boldsymbol{f}$ and the Dirichlet boundary condition are computed by using the chosen exact solution. We have shown 
the errors in the velocity and pressure approximating using norms as in the previous example in Table 2. We can see that the velocity approximation converges linearly to the exact solution, whereas the pressure approximation shows a super-convergence as in the first example.

Table 2: Discretization errors for the velocity and pressure, Example 2

\begin{tabular}{|c|c|c|c|c|c|}
\hline level $l$ & \# elem. & \multicolumn{2}{|c|}{$\left|u-u_{h}\right|_{1, h}$} & \multicolumn{2}{c|}{$\left\|p-p_{h}\right\|_{0, \Omega}$} \\
\hline 0 & 8 & $4.85293 \mathrm{e}+00$ & & $3.78781 \mathrm{e}+00$ & \\
\hline 1 & 64 & $2.57400 \mathrm{e}+00$ & 0.91 & $1.36397 \mathrm{e}+00$ & 1.47 \\
\hline 2 & 512 & $1.31776 \mathrm{e}+00$ & 0.97 & $4.77524 \mathrm{e}-01$ & 1.51 \\
\hline 3 & 4096 & $6.65496 \mathrm{e}-01$ & 0.99 & $1.66988 \mathrm{e}-01$ & 1.52 \\
\hline 4 & 32768 & $3.34230 \mathrm{e}-01$ & 0.99 & $5.85231 \mathrm{e}-02$ & 1.51 \\
\hline 5 & 262144 & $1.67459 \mathrm{e}-01$ & 1.00 & $2.05565 \mathrm{e}-02$ & 1.51 \\
\hline
\end{tabular}

\section{Conclusion}

We have presented a finite element method for the Stokes equations using lowest order Crouzeix-Raviart element for the velocity and continuous linear element for the pressure. We have proved the fact the stabilization is not necessary as proposed in [10]. The numerical experiments support the theoretical results.

\section{References}

[1] P.B. Bochev, C.R. Dohrmann, and M.D. Gunzburger. Stabilization of low-order mixed finite elements for the Stokes equations. SIAM Journal on Numerical Analysis, 44:82-101, 2006.

[2] S.C. Brenner and L.R. Scott. The Mathematical Theory of Finite Element Methods. Springer-Verlag, New York, 1994.

[3] F. Brezzi and M. Fortin. Mixed and hybrid finite element methods. Springer-Verlag, New York, 1991.

[4] P.G Ciarlet. The Finite Element Method for Elliptic Problems. North Holland, Amsterdam, 1978. 
[5] M. Crouzeix and P.A. Raviart. Conforming and nonconforming finite element methods for solving the stationary stokes equations. RAIRO Anal. Numér., 7:33-36, 1973.

[6] R. E. Ewing, T. Lin, and Y. Lin. On the accuracy of the finite volume element method based on piecewise linear polynomials. SIAM Journal on Numerical Analysis, 39:1865-1888, 2002.

[7] V. Girault and P.-A. Raviart. Finite Element Methods for Navier-Stokes Equations. Springer-Verlag, Berlin, 1986.

[8] B.P. Lamichhane. A mixed finite element method based on a biorthogonal system for nearly incompressible elastic problems. In Geoffry N. Mercer and A. J. Roberts, editors, Proceedings of the 14th Biennial Computational Techniques and Applications Conference, CTAC-2008, volume 50 of ANZIAM J., pages C324-C338, 2008.

[9] B.P. Lamichhane. Inf-sup stable finite element pairs based on dual meshes and bases for nearly incompressible elasticity. IMA Journal of Numerical Analysis, 29:404-420, 2009.

[10] J. Li and Z. Chen. A new local stabilized nonconforming finite element method for the Stokes equations. Computing, 82:157-170, 2008. 